\title{
IMPACT OF COVID-19 ON MENTAL HEALTH OF HEALTHCARE WORKERS DURING PANDEMIC; AN EXPERIENCE AT A TERTIARY CARDIAC CARE SET UP
}

\author{
Sajida Parveen, Sajal Awan*, Faiza Rabbani**, Farrah Pervaiz, Aleena Khan, Fahad Ahmad Khan ${ }^{* * *}$, Rehana Javaid, \\ Naheed Kausar****
}

Armed Forces Institute of Cardiology/National Institute of Heart Disease (AFIC/NIHD)/National University of Medical Sciences (NUMS) Rawalpindi Pakistan, *Rawal Dental College Islamabad Pakistan, ${ }^{* *}$ Institute of Public Health Lahore Pakistan, ${ }^{* * *}$ Pak Emirates Military Hospital/National University of Medical Sciences (NUMS) Rawalpindi Pakistan, ${ }^{* * * *}$ HQ AAD Rawalpindi Pakistan

\begin{abstract}
Objective: To assess the frequency of mental health problems among healthcare workers related to COVID-19 pandemic among health care workers in a tertiary cardiac care center.

Study Design: Descriptive cross sectional study.

Place and Duration of Study: This study was conducted at Armed Forces Institute of Cardiology/ National Institute of Heart Diseases (AFIC/NIHD) Rawalpindi (Pakistan) from Apr 2020 to Jun 2020.

Methodology: This study was a hospital-based cross-sectional study consisted of 100 healthcare workers including 50 nurses and 50 doctors who volunteered to enroll in our study were included. Approval from the clinical research ethics committee of cardiac care hospital was received before the initiation of this study. Verbal informed consent was provided by all study participants prior to their enrollment. Exclusion criteria comprised of the healthcare workers who refused to participate in it. This study followed the COVID-19 Pandemic Mental Health Questionnaire (CoPaQ) 14. Descriptive statistics were used to describe the percentages and frequencies. Descriptive statistics were used to describe the percentages and frequencies were calculated and analyzed using the Statistical Package for the Social Sciences (SPSS), version 23.0.

Results: A total of 100 healthcare workers completed the survey. Mean age of the healthcare workers was $36.5 \pm$ 27.0 years (median $=36.00)$, and $75(75 \%)$ were women and men comprised $25(25 \%)$. Of all participants, $50(50.0 \%)$ were nurses, and $50(50 \%)$ were doctors. A considerable proportion of participants reported symptoms of COVID$19,6(6.0 \%)$, tested positive $14(14 \%)$, currently quarantine $24(24.0 \%)$. While 12 healthcare workers experienced 14 day quarantine time period. 16 healthcare workers reported COVID test positive of their closed one. The fear of infecting others with COVID-19 was found to be $30(30 \%)$. The mental stress that that COVID-19 will personally affect us was found in 28 (28\%) of healthcare workers. The fear that consequences of the COVID-19 will be severe health disasters was found in $40(40 \%)$ of the individuals.

Conclusion: In this study of physicians and nurses in hospitals with wards for patients with COVID-19, health care workers responding to the spread of COVID-19 reported high rates of symptoms of anxiety, fear, and distress.
\end{abstract}

Keywords: COVID-19, Healthcare workers, Quarantine.

This is an Open Access article distributed under the terms of the Creative Commons Attribution License (http://creativecommons.org/licenses/by/4.0), which permits unrestricted use, distribution, and reproduction in any medium, provided the original work is properly cited.

\section{INTRODUCTION}

In December 2019, a new severe type of pneumonia, later known as novel coronavirus disease (COVID-19), was recounted in Wuhan, Hubei, China. Over the subsequent months, it rapidly prolonged around the world although not all countries have been likewise affected. By 24 May 2020, the World Health Organization (WHO) had been up-to-dated of 5,165,481 cases

Correspondence: Ms Sajida Parveen, Department of Cardiology, AFIC/ NIHD, Rawalpindi Pakistan of COVID-19, including 336,430 deaths ${ }^{1}$. Earlier research on other infectious diseases, including the Severe Acute Respiratory Syndrome (SARS), the Middle East respiratory syndrome (MERS) and the Ebola virus disease, steadily showed that many healthcare professionals (HPs) reported symptoms of anxiety and depression, both during and after the outbreak, causing a severe impact on their coping abilities, in some cases with long-lasting effects 2,3 . In many countries, especially in those that had not experienced recent epidemic outbursts, Health care workers have 
been opposed to unfore seen painful practices during the COVID-19 pandemic ${ }^{4,5}$.

Health care workers employed in the first line of care, with advanced clinical duties and those who have been infected have had higher prevalence of anxiety and depressive symptoms 6-13.

Fronting this critical circumstances, health care workers on the front line who are directly involved in the treatment, diagnosis, and care of patients with COVID-19 are at risk of increasing psychological distress and other mental health symptoms. The ever-increasing number of confirmed and suspected cases, devastating workload, reduction of personal protection equipment, coverage of general media, deficiency of specific drugs, and outlooks of being imperfectly strengthened may all add to the mental burden of these health care workers. Previous studies have reported adverse psychological reactions to the 2003 SARS outbreak among health care workers 14-17. Researches revealed that those health care workers feared contagion and infection of their family, friends, and colleagues, sens of ambiguity and stigmatization ${ }^{14,15}$ described averseness to work or anticipating resignation, and stated under going high levels of stress, anxiety, and depression symptoms ${ }^{18}$, which could have resulted in long-term psychological associations. Similar apprehensions about the mental health, psychological adjustment, and recovery of health care workers treating and caring for patients with COVID-19 are now arising.

The aim of this study was to evaluate the current evidence based information about the impact of the COVID-19 pandemic on on the mental health of health care workers in a tertiary cardiac care center.

To evaluate the impact of the COVID-19 pandemicon on the mental health of health care workers in a tertiary cardiac care center.

\section{METHODOLOGY}

This study followed the COVID-19 Pandemic Mental Health Questionnaire (CoPaQ) ${ }^{19}$, which is a newly developed and highly comprehensive self-report measure of personal and social consequences of the COVID-19-pandemic with an application scope world-wide. The questionnaire covers a wide range of areas of interest affected by the COVID-19 pandemic; that is, COVID-19 infection status, socio-demographic background, and the impact on risk perception, affect, thoughts, behavior, mental health, media usage, institutional trust and social cohesion. It took around 20 minutes to complete. Approval from the clinical research ethics committee of cardiac care hospital wasreceived before the initiation of this study. Verbal informed consent was provided by all studyparticipants prior to their enrollment. This study was a cross-sectional, hospital-based consisted of 100 healthcare workers including 50 nurses and 50 doctors who volunteered to enroll in our study were included.

\section{RESULTS}

A total of 100 healthcare workers completed the survey. Mean age of the healthcare workers was $36.5 \pm 27$ years (median=36.00), and $75(75 \%)$ were women and men comprised $25(25 \%)$. Of allparticipants, $50(50.0 \%)$ were nurses, and 50 (50\%) were doctors.

A considerable proportion of participants reported symptoms of COVID-19 6 (6.0\%), tested positive $14(14 \%)$, currently quarantine 24 (24\%). While 12 healthcare workers experienced 14 day quarantine time period ${ }^{16}$. Healthcare workers reported COVID test positive of their closed one. On the other hand, 36 reported death of their closed ones due to COVID-19. Risk factors for COVID were present in age great than 60 years 10 (10\%), CVD 6 (6\%), Immunodeficiency 6 (6\%). Nurses, women, frontline health care workers, and those working in critical care units reported more severe degrees of all measurements of mental health symptoms than other health care workers.

On the Likert scale of risk perception of COVID-19 pandemic questionnaire, 30.0\% healthcare workers experienced that there are no means of control over the pandemic worried while 26 
(26\%) experienced that they will not infect themselves with COVID-19. Thirty (30\%) showed their recommended by 60 (60\%). Only 34 (34\%) agreed to donate blood in this pandemic.

Table-I: Common fears regarding COVID-19.

\begin{tabular}{c|l|c}
\hline S. No. & Fear of Uncontrollable Pandemic & $\mathbf{3 0 ~ ( 3 0 \% )}$ \\
\hline 1. & Fear of Infecting themselves & $26(26 \%)$ \\
\hline 2. & Fear of Infecting others & $30(30 \%)$ \\
\hline 3. & Fear of severe health disaster & $40(40 \%)$ \\
\hline 4. & Fear of loosing their loved ones & $42(42 \%)$ \\
\hline Table-II: Behavior of Health Care Workers Regarding Usefulness of Basic Hygienic Measures. \\
\hline S. No. & Social Distancing & $\mathbf{8 0 ~ ( 8 0 \% )}$ \\
\hline 1. & Coughing or sneezing into crook of arm & $70(70 \%)$ \\
\hline 2. & Avoid touching face & $74(74 \%)$ \\
\hline 3. & Regular hand wash & $84(84 \%)$ \\
\hline 4. & Washing hands for 30 seconds & $86(86 \%)$ \\
\hline 5. & Disinfection of hands and objects & $86(86 \%)$ \\
\hline Table-III: Reduction of Social Contacts & $\mathbf{7 4 ( 7 4 \% )}$ \\
\hline S. No. & Cancelling Private Meeting and Visits & $70(70 \%)$ \\
\hline 1. & Cancelling trips to other cities & $78(78 \%)$ \\
\hline 2. & Avoiding restaurants & $86(86 \%)$ \\
\hline 3. & Avoiding shaking hands & $40(40 \%)$ \\
\hline 4. & Making work from home & \\
\hline
\end{tabular}

fear that they will infect others with COVID19. Twenty Eight $(28 \%)$ of healthcare workers showed mental stress that that COVID-19 will personally affect us. Fourty (40\%) of the individuals had shown their fear that consequences of the COVID-19 will be severe health disasters. Fifty $(50 \%)$ of the HC workers had shown that they will not die of COVID-19, while opposite statement was recorded by $8(8 \%)$. Forty two $(42 \%)$ of the individuals had fear that they will loose their loved ones due to COVID-19 as shown in table-I. Healthcare workers' behavior regarding basic hygienic measures is recorded and given in table-II.

Building up of stocks was also observed in healthcare workers during this pandemic. Thirty eight $(38 \%)$ preferred to stock detergents, soaps and washing products. Only 34 (34\%) individuals showed the interest of storing water and food products. Toilet paper storage was seen in $38(38 \%)$. Stock up of cash was observed in only $36(36 \%)$ healthcare workers. Regarding political measures like closure of educational institutions, borders, playgrounds, was strongly

\section{DISCUSSION}

The COVID-19 has disputed many healthcare workers with unforeseen, life threatening experiences for which they had not been qualified. Although they are used to perceiving trauma and to regularly dealing with loss ${ }^{26}$, the high morbidity and mortality rates of this pandemic, the shortage in personal protective equipment, the fear of they or their family members becoming infected, the absence of an effective treatment /vaccine on the immediate limit and the new restraining public health policies initiated in most countries, have altered their normal scenario.

Therefore, during the pandemic, unpleasant emotions, including fear, hyper-arousal, intrusive memories and insomnia, as well as some related to sadness or emotional exhaustion were experienced by majority of them. The more they were exposed to unexpected life-threatening circumstances or uncertainty, the more mental distress they were expected to experience.

During the COVID-19 pandemic, many healthcare workers have been infected, requiring to be quarantined or even hospitalized. From pre- 
vious experiences, we learnt that those quarantined have a tendency to sense more fearful, frustrated, deserted and isolated than non-healthcare workers ${ }^{21}$.

In fact, a big alarm is that health care workers may be unwilling to ask for help if needed. Self-treatment, denial, rationalization or minimization may be initial defense mechanisms used to challenge stressful circumstances but may result in not pursuing suitable help when developing a mental disorder. During the COVID-19 outbreak, this propensity might have transformed ${ }^{22}$. The social recognition health care workers are getting during this pandemic together with the mass and social media diffusion of their testimonies could help to worse the internal psychological barriers to seeking professional support if necessary. Psychological assistance delivered to health care workers during the pandemic should be prolonged afterwards for cases at higher risk of evolving mental disorders ${ }^{23}$.

Longitudinal studies should help determine the long-lasting magnitudes of the acute distress among the health care workers facing this pandemic. Regardless of the importance of quantitative analysis, qualitative research may subsidize to draw a more accurate picture of individuals and socio-cultural narratives related to the pandemic and their influence on trauma response as we already know that providing a meaning to traumatic experiences and having social support connects with a lower presence of mental distress in the short and mid-to-long term.

\section{CONCLUSION}

In this study of physicians and nurses in hospitals with wards for patients with COVID-19, health care workers responding to the spread of COVID-19 reported high rates of symptoms of anxiety, fear, and distress. Protecting health care workers is an important component of public health measures for addressing the COVID-19 epidemic.

\section{CONFLICT OF INTEREST}

This study has no conflict of interest to be declared by any author.

\section{REFERENCES}

1. World Health Organization (WHO) Coronavirus Disease (COVID-19) Dashboard. Available from: https://covid19. who.int/?gclid¹/4CjwKCAjw_LL2BRAkEiwAv2Y3SekyJ8kMY7y LRUzWfcdLLQPJI

BpwfUFFsJWPG8GCQqYTp0fEY9MvKxoCCxgQAvD_BwE

2. Go'mez-Duran EL, Martin-Fumado' C, GarcíaForero C. The psychological impact of quarantine on healthcare workers. Occup Environ Med 2020; 77(10): 1-5.

3. Bettinsoli ML, Di Riso D, Napier JL, Moretti L, Bettinsoli PF, Deimedico M, et al. Psychological impact and contextual factors ssociated with physical and mental health conditions of Italian Healthcare Professionals during the COVID-19 disease outbreak. PsyArXiv 2020. Available from: https://psyarxiv.com /w89fz/

4. Chen Q, Liang M, Li Y, Guo J, Fei D, Wang L, et al. Mental health care for medical staff in China during the COVID-19 outbreak. Lancet Psychiatry 2020; 7(1): e15-16.

5. Blanco-Donoso LM, Garrosa E, Moreno-Jiménez J, Ga' lvezHerrer M, Moreno-Jiménez B. Occupational psychosocial risks of health professionals in the face of the crisis produced by the COVID-19: from the identification of these risks to immediateaction. Int J Nurs Stud 2020; 2(1): 100003.

6. Xue-Hui F, Wu L, Lun-Shan L, Kan X-H, Wang H, Yang-jun X. S Analysis of mental health status and needs of health care workers in designated medical institutions of tuberculosis during the epidemic period of COVID-19. Res Square 2020; 1(1): 1-4.

7. Lu W, Wang H, Lin Y, Li L. Psychological status of medical workforce during the COVID-19 pandemic: a cross-sectional study. Psychiatry Res 2020; 288(1): 1129-36.

8. Liu X, Liang S, Zhang R, Wei Y, Li J, Wang C, et al. Perceived social support and its impact on psychological status and quality of life of medical staffs after outbreak of SARS-CoV-2 pneumonia: a cross-sectional study. Lancet 2020; 1(1): 1-6.

9. Lai J, Ma S, Wang Y, Cai Z, Hu J, Wei N, et al. Factors associated with mental health outcomes among health care workers exposed to coronavirus disease 2019. J Am Med Assoc 2020; 3e: 203976.

10. Xiao H, Zhang Y, Kong D, Li S, Yang N. The effects of social support on sleep quality of medical staff treating patients with coronavirus disease 2019 (COVID-19) in January and February 2020 in China. Med Sci Monit 2020; 26(1): e923549.

11. Dai Y, Hu G, Xiong H, Qiu H, Yuan X. Psychological impact of the coronavirus disease 2019 (COVID-19) outbreak on healthcare workers in China. Lancet Psychiatr 2020; 20030874.

12. Cai H, Tu B, Ma J, Chen L, Fu L, Jiang Y, et al. Psychological impact and coping strategies of frontline medical staff in Wuhan between January and March 2020 during the outbreak of coronavirus disease 2019 (COVID-19) in Hubei, China. Me Sci Monit 2020; 26(1): e924171.

13. Mo Y, Deng L, Zhang L, Lang Q, Liao C, Wang N, et al. Work stress among Chinese nurses to support Wuhan in fighting against COVID-19 epidemic. J Nurs Manag 2020; 1(1): $1-8$.

14. Maunder R, Hunter J, Vincent L. The immediate psychological and occupational impact of the 2003 SARS outbreak in a teaching hospital. C MAJ 2003; 168(10): 1245-51.

15. Bai Y, Lin CC, Lin CY, Chen JY, Chue CM, Chou P. Survey of stress reactions among health care workers involved with the SARS outbreak. Psychiatr Serv 2004; 55(9): 1055-57.

16. Lee AM, Wong JG, McAlonan GM,. Stress and psychological distress among SARS survivors 1 year after the outbreak. Can J Psychiatr 2007; 52(4): 233-40. 
17. Chua SE, Cheung V, Cheung C. Psychological effects of the SARS outbreak in Hong Kong on high-risk health care workers. Can J Psychiatry 2004; 49(6): 391-93.

18. COVID-19 Pandemic Mental Health Questionnaire (CoPaQ): Rek S, Freeman D. The COVID-19 pandemic mental health questionnaire $(\mathrm{CoPaQ})$ : Int-roducing a comprehensive measure of the psychosocial impact of the coronavirus crisis. 2020; 1(1): 1-5.

19. Gerada C. Doctors and their defences. Bio Mrd J 2019; 364(2): $871-85$.

20. Lu W, Wang H, Lin Y, Li L. Psychological status of medical workforce during the COVID-19 pandemic: a cross-sectional study. Psychiatr Res 2020; 288(4): 1129-36.

21. Wu P, Fang Y, Guan Z, Fan B, Kong J, Zhongling Y, et al. The psychological impact of the SARS epidemic on hospital employees in China: exposure, risk perception, and altruistic acceptance of risk. Can J Psychiatr 2009; 54(1): 302-11.

22. Rimmer A, Chatfield C. What organisations around the world are doing to help improve doctors' wellbeing. Bio Med J 2020; 369(1): m1541.

23. Braquehais MD, Sher L. Posttraumatic stress disorder in warve terans: a discussion of the neuroevolutionary time-depth principle. J Affect Disord 2010; 125(1): 1-9. 